\title{
Lapatinib-capecitabine versus capecitabine alone as radiosensitizers in RAS wild-type resectable rectal cancer, an adaptive randomized phase II trial (LaRRC trial): study protocol for a randomized controlled trial
}

Nuno Sousa ${ }^{1 *}$, Olga Sousa ${ }^{2}$, Lúcio Lara Santos ${ }^{3}$, Rui Henrique ${ }^{4,5}$, Manuel R. Teixeira ${ }^{6,7}$, Mário Dinis-Ribeiro ${ }^{8,9}$ and Armando Teixeira-Pinto ${ }^{10}$

\begin{abstract}
Background: Preoperative radiochemotherapy followed by surgical removal of the rectum with total mesorectum excision is the preferred treatment option for stages II and III rectal cancer. However, development of metastatic disease is the main cause of death for these patients with 5-year disease-free survival rates of $56 \%$. Anti-epidermal growth factor receptor (EGFR) targeted therapy is effective in metastatic rectal cancer, and human epidermal growth factor receptor 2 (HER-2) signaling may mediate resistance to EGFR inhibitors. Moreover, preclinical data support a synergistic effect of EGFR inhibition with radiation therapy.
\end{abstract}

Methods/design: This Bayesian phase II trial with adaptive randomization was designed to assess the efficacy of adding lapatinib, a dual inhibitor of EGFR and HER-2, to standard radiochemotherapy with capecitabine in stages II and III rectal cancer.

Discussion: The results of this trial will provide evidence of the feasibility and efficacy of the combination of lapatinib-capecitabine as radiosensitizers and explore potential predictive biomarkers for response to this novel neoadjuvant approach to resectable rectal cancer.

Trial registration: EudraCT 2013-001203-36. Registered on 13 December 2013.

Keywords: Rectal neoplasms, Radiotherapy, Capecitabine (capecitabine [supplementary concept]), Lapatinib (lapatinib [supplementary concept]), Neoadjuvant therapy, Clinical trial, Phase II, Randomized adaptive trial, Bayesian adaptive randomization

\footnotetext{
* Correspondence: nuno.sousa@ipoporto.min-saude.pt

1 Medical Oncology Department, IPO Porto FG, EPE, Rua António Bernardino

de Almeida, 4200-072 Porto, Portugal

Full list of author information is available at the end of the article
} 


\section{Background}

Colorectal cancer is the third most incident cancer and the second most common cause of cancer death in Europe [1]. Cancer of the rectum accounts for a third of colorectal cancer cases [2] with 5-year survival rates ranging from $28-55 \%$ in men and 30-64\% in women [3].

Current treatment options for patients with rectal cancer include surgery, radiation therapy, chemotherapy, and targeted systemic therapy. The choice of therapy depends on disease stage, overall health status, and patient preferences. For localized disease, surgical removal of the rectum with total mesorectum excision is the surgical technique of choice, with an estimated overall survival of $86 \%$ and a local recurrence rate of $9 \%$ at 2 years [4]. However, the use of radiotherapy before surgical resection decreases local recurrence rate and impacts overall survival [5]. Moreover, the addition of fluoropyrimidine-based chemotherapy to preoperative radiotherapy further improves local control and pathologic response rates [6]. In patients treated with preoperative radiochemotherapy, whenever downstaging occurs, the risk of death due to cancer is significantly reduced [7]. Despite these therapeutic improvements, patients with localized rectal cancer have 5-year diseasefree and overall survival rates of about $59 \%$ and $67 \%$, respectively [8, 9]. For these patients, development of metastatic disease is the main cause of death.

Capecitabine is a non-cytotoxic fluoropyrimidine carbamate, which functions as an orally administered precursor of the cytotoxic agent 5-fluorouracil. Capecitabine is active as a single agent in colorectal cancer, and it is approved for the adjuvant and palliative treatment of patients with colorectal cancer [10]. Its value as a radiosensitizer in rectal cancer has been established by several phase II trials, with pathologic response rates that range from 7-38\% [11-15]. In a phase III non-inferiority trial, radiochemotherapy with capecitabine was shown to be non-inferior to 5 -fluorouracil radiochemotherapy, with survival rates of $76 \%$ at 5 years [16]. Taken together, these data and the more comfortable administration regimen of capecitabine compared to infusional 5-fluorouracil make it the preferential agent for the neoadjuvant treatment of patients with rectal cancer.

Colorectal cancer frequently overexpresses epidermal growth factor receptor (EGFR) [17]. Ligand binding to the extracellular portion of the receptor promotes homodimerization or heterodimerization and subsequent activation of the receptor. This leads to increased cell growth, proliferation, and mobility, decreased apoptosis, and stimulation of angiogenesis. Currently available drugs targeting the EGFR include monoclonal antibodies (e.g., cetuximab and panitumumab) and small molecule tyrosine kinase inhibitors (e.g., gefitinib, erlotinib, and lapatinib). In metastatic colorectal cancer, cetuximab and panitumumab were shown to be clinically effective
[18-23]. Resistance to anti-EGFR antibodies may be mediated by HER-2, since the addition of anti-HER-2 targeting agents to cetuximab is able to overcome this resistance $[24,25]$. These data support the development of therapeutic strategies targeting both EGFR and HER2 in colorectal cancer. Additionally, targeting the EGFR pathway during neoadjuvant radiochemotherapy is associated with an increased number of cells in the G1 phase of the cell cycle (the most radiosensitive phase) and increased apoptosis, and prevents repair of DNA damage induced by radiation exposure, thus suggesting a possible synergistic effect of EGFR inhibition with radiation therapy [26, 27]. This strategy has been explored with cetuximab and gefitinib in combination with fluoropyrimidine-based radiochemotherapy in the phase I/II setting with disappointing results [28-30]. However, all these studies were conducted before the predictive role of RAS mutations in anti-EGFR treated patients was recognized; therefore, they may have been underpowered to detect a clinically relevant improvement for this therapeutic strategy.

Lapatinib is a small molecule dual tyrosine kinase inhibitor targeting both EGFR and HER-2. Preclinical studies have shown that lapatinib is active in colorectal cancer cell lines [31, 32], and its radiosensitizing properties have also been demonstrated in preclinical models $[33,34]$. In treating metastatic colorectal cancer, lapatinib has been shown to be well tolerated, both as a single agent and in combination with chemotherapy, but with limited activity [35-38]. However, no population enrichment strategy based on tumor characteristics was implemented, and these trials may have been underpowered to identify any clinically relevant activity. Considering the clinical value of anti-EGFR therapy in RAS wild-type colorectal cancer [19-23, 39] and the theoretical advantage of anti-EGFR treatment during radiotherapy [26, 27] together with the data suggesting that dual inhibition of EGFR and HER-2 may be synergistic [31, 32], one can hypothesize that lapatinib is an ideal candidate for optimizing the neoadjuvant treatment of rectal cancer.

A randomized phase II trial with an adaptive Bayesian randomization design is proposed to assess the efficacy and safety of the tyrosine kinase inhibitor lapatinib combined with the fluoropyrimidine analog capecitabine as radiosensitizers in patients with clinical stage II/III resectable rectal cancer without activating RAS mutations.

\section{Methods/design}

This single-center phase II trial is designed with an adaptive Bayesian randomization, which will allocate eligible patients to either lapatinib-capecitabine ( $\operatorname{arm~A}$ ) or capecitabine (arm B) during standard radiation treatment for rectal cancer. The first 20 patients will be allocated at a 
1:1 ratio. From that time point onward the randomization of a patient to either arm will be weighted by the updated estimate of the success probability in each arm, hence giving patients a higher chance of being allocated to the current most efficacious arm of the trial. The trial is designed to be stopped earlier if evidence of superiority of one arm over the other, futility, or unacceptable toxicity in arm $\mathrm{A}$ is identified.

\section{Population and setting}

The trial will be implemented at Instituto Português de Oncologia do Porto Francisco Gentil, EPE, where patients will be considered eligible for the trial based on the following criteria.

\section{Inclusion criteria}

Each patient must meet all of the following inclusion criteria to be enrolled in the study:

1. Age of 18 years or above

2. Diagnosis of adenocarcinoma of the rectum with the primary malignant lesion located between the dentate line up to $10 \mathrm{~cm}$ of the anal verge by endoscopic examination

3. Absence of activation mutation in KRAS exons 2 (codons 12 and 13), 3 (codons 59 and 61), and 4 (codons 117 and 146) and NRAS exons 2 (codons 12 and 13), 3 (codons 59 and 61), and 4 (codons 117 and 146)

4. Clinical stage II or III disease, according to American Joint Committee on Cancer Staging classification, 7th edition [40]. For clinical staging, the following procedures must have been conducted within 4 weeks of treatment allocation:

(a) Clinical history and physical examination

(b) Endoscopic ultrasound of the primary lesion

(c) Thoracic, abdominal, and pelvic computed tomographic (CT) scan

5. Clinically judged to be able to undergo curative resection of the rectal neoplasm despite preoperative radiochemotherapy

6. Clinically judged to be able to undergo pelvic radiation therapy to a total dose of $50.4 \mathrm{~Gy}$

7. Provision of signed informed consent

\section{Exclusion criteria}

Patients meeting any of the following exclusion criteria are not to be enrolled in the study:

1. Absence of baseline histological sample of the primary tumor

2. Pregnant or lactating women

3. Unwillingness or inability to comply with effective contraception, if the patient is fertile.
4. Impaired renal function defined as creatinine clearance $<60 \mathrm{~mL} / \mathrm{min}$ according to the Cockcroft-Gault formula

5. Impaired hematological function as defined by any of the following on pretreatment evaluation:

(a) Hemoglobin concentration $<10.0 \mathrm{~g} / \mathrm{dL}$,

(b) Absolute neutrophil count $<1500 / \mu \mathrm{L}$

(c) Platelet count $<100,000 / \mu \mathrm{L}$

6. Impaired hepatic function defined by any of the following on pretreatment evaluation:

(a) Serum level of aspartate aminotransferase $>1.5 \times$ ULN,

(b) Serum level of alanine aminotransferase $>1.5 \times$ ULN,

(c) Serum level of alkaline phosphatase $>1.5 \times$ ULN,

(d) International normalized ratio $>1.5$

(e) Serum concentration of total bilirubin $>1.5 \times \mathrm{ULN}$

7. Symptomatic heart failure or a left ventricular ejection fraction (LVEF) below the institution's lower limit of normality (LLN) as assessed through equilibrium radionuclide angiography

8. Known intolerance to any of the study drugs.

9. Known dihydropyrimidine dehydrogenase (DPD) deficiency

10. Concurrent treatment with CYP3A4 inducers (as detailed in the following section)

11. Concurrent treatment with CYP3A4 inhibitors (as detailed in the following section)

12. Likely inability to comply with the protocol or cooperate fully with the investigator and site personnel

13. Current enrollment in another clinical trial.

\section{Trial procedures and study interventions}

Recruitment and enrollment for this study will be from the institution's local practice or referrals from other physicians. All trial-related procedures and interventions will be performed according to the predefined schedule of events (Table 1). Before any study-related procedure, each patient will provide written informed consent, unless that procedure was performed as part of the patient's standard medical care and is eligible for trial enrollment. Prior to study treatment a screening period, no longer than 28 days, will be considered. During this period, eligibility will be assessed and tumor tissue procured for biomarker analysis. Eligible patients will be randomly allocated to an experimental arm (arm A), which will test the addition of lapatinib to radiochemotherapy with capecitabine or a control arm (arm B), which will consist of standard radiochemotherapy with capecitabine. Patients allocated to arm A will undergo an initial lead-in period of 14 days with lapatinib before radiochemotherapy. At the end of the lead-in period, 
Table 1 Schedule of events

\begin{tabular}{|c|c|c|c|c|c|c|c|c|c|c|c|c|c|}
\hline & \multirow[t]{2}{*}{ Screening } & \multirow{2}{*}{$\begin{array}{l}\text { Random } \\
\text { allocation () }\end{array}$} & \multicolumn{8}{|c|}{ Treatment period } & \multirow{2}{*}{$\begin{array}{l}\text { EOT } \\
\text { Day } \\
66 \pm 3 \text { days }\end{array}$} & \multirow{2}{*}{$\begin{array}{l}\text { Surgery } \\
\text { Day } \\
87-94\end{array}$} & \multirow{2}{*}{$\begin{array}{l}\text { EOS } \\
28(+7) \text { days } \\
\text { after surgery }\end{array}$} \\
\hline & & & Day -14(b) & Day -7 & Day $1\left({ }^{(}\right)$ & $\begin{array}{l}\text { Day } \\
8 \pm 3 \text { days }\end{array}$ & $\begin{array}{l}\text { Day } \\
15 \pm 3 \text { days }\end{array}$ & $\begin{array}{l}\text { Day } \\
22 \pm 3 \text { days }\end{array}$ & $\begin{array}{l}\text { Day } \\
29 \pm 3 \text { days }\end{array}$ & $\begin{array}{l}\text { Day } \\
36 \pm 3 \text { days }\end{array}$ & & & \\
\hline Informed consent & $x$ & & & & & & & & & & & & \\
\hline Inclusion/exclusion criteria & X & $x$ & & & & & & & & & & & \\
\hline Demographics & $x$ & & & & & & & & & & & & \\
\hline Medical history & $x$ & & & & & & & & & & & & \\
\hline Concomitant medications & $x$ & $x$ & & $x$ & $x$ & $x$ & $x$ & $x$ & $x$ & $x$ & $x$ & & $x$ \\
\hline Adverse events & & & & $x$ & $x$ & $x$ & $x$ & $x$ & $x$ & $x$ & $x$ & & $x$ \\
\hline Physical examination & $x$ & $x$ & & & & & & & & & & & \\
\hline Vital signs & $x$ & $x$ & & $x$ & $x$ & $x$ & $x$ & $x$ & $x$ & $x$ & $x$ & & $x$ \\
\hline Weight & $x$ & $x$ & & $x$ & $x$ & $x$ & $x$ & $x$ & $x$ & $x$ & $x$ & & $x$ \\
\hline Height & $x$ & & & & & & & & & & & & \\
\hline ECOG performance status & $x$ & $x$ & & $x$ & $x$ & $x$ & $x$ & $x$ & $x$ & $x$ & $x$ & & $x$ \\
\hline Electrocardiogram (12-lead) & $x$ & & & & & & & & & & $x$ & & $x$ \\
\hline MUGA & $x$ & & & & & & & & & & $x$ & & $x$ \\
\hline Hematology & $x$ & $x$ & & $x$ & $x$ & $x$ & $x$ & $x$ & $x$ & $x$ & $x$ & & $x$ \\
\hline Serum chemistries & $x$ & $x$ & & $x$ & $x$ & $x$ & $x$ & $x$ & $x$ & $x$ & $x$ & & $x$ \\
\hline Hemostasis & $x$ & & & & & & & & & & & & \\
\hline Pregnancy test & $x$ & & & & & & & & & & $x$ & & $x$ \\
\hline $\begin{array}{l}\text { CT - scan } \\
\text { (chest, abdomen, pelvis) }\end{array}$ & $x$ & & & & & & & & & & & & \\
\hline Endoscopic ultrasound & $x$ & & & & & & & & & & & & \\
\hline Tumor sampling & $x$ & & & & $X\left({ }^{c}\right)$ & & & & & & & $x$ & \\
\hline Lapatinib (arm A) & & & $1250 \mathrm{mg} / \mathrm{d}$ & until the & last day of & adiotherapy & & & & & & & \\
\hline $\begin{array}{l}\text { Capecitabine } \\
\text { (arms A and B) }\end{array}$ & & & & & $825 \mathrm{mg} / \mathrm{n}$ & 2 twice daily & from first to $\mid$ & ast day of rad & therapy & & & & \\
\hline Radiation therapy & & & & & 50.4 Gy in & 28 fractions & of $1.8 \mathrm{~Gy}, 5 \mathrm{da}$ & ys per week & & & & & \\
\hline
\end{tabular}

EOT end of treatment visit, EOS end of study visit

(a) Within 4 weeks of screening visit

(b) Not more than 3 days before day -14 in arm A or more than 14 days before day 1 in arm B. In arm B, random allocation visit procedures are valid for day 1 visit if $<7$ days have elapsed

(c) Arm A only: to be performed within a 3-day time window of day 1 of radiation treatment 
patients in arm A will have a tumor biopsy performed for biomarker assessment. As lapatinib is a substrate for CYP3A4, inducers and inhibitors of this enzyme are prohibited from screening through discontinuation from study, and a predefined wash-out period prior to random allocation has been set (Table 2). Seven to eight weeks after completion of radiochemotherapy, patients will be surgically treated. For the duration of the trial, no other concurrent anticancer therapy or investigational agents are allowed. During the trial, neither patients nor treating physician will be blinded to treatment interventions.

\section{Lapatinib}

Lapatinib, 250-mg tablets, will be self-administered, on an empty stomach (either $1 \mathrm{~h}$ before or after a meal), with water, once daily at approximately the same time each day, at a dose of $1250 \mathrm{mg} / \mathrm{d}$. Treatment with

Table 2 CYP3A4 inducers and inhibitors

\begin{tabular}{|c|c|c|}
\hline Drug class & Agent & Wash-out \\
\hline \multicolumn{3}{|l|}{ CYP3A4 inducers } \\
\hline Antibiotics & $\begin{array}{l}\text { All rifamycin agents } \\
\text { (e.g., rifampicin, rifabutin, } \\
\text { rifapentine) }\end{array}$ & 14 days \\
\hline Anticonvulsants & $\begin{array}{l}\text { Phenytoin, carbamazepine, } \\
\text { barbiturates (e.g., phenobarbital) }\end{array}$ & \\
\hline Antiretrovirals & Efavirenz, nevirapine & \\
\hline $\begin{array}{l}\text { Glucocorticoids } \\
\text { (oral) }\end{array}$ & $\begin{array}{l}\text { Cortisone (>50 mg), hydrocortisone } \\
(>40 \mathrm{mg}) \text {, prednisone }(>10 \mathrm{mg}) \\
\text { methylprednisone }(>8 \mathrm{mg}) \\
\text { dexamethasone }(>1.5 \mathrm{mg})\end{array}$ & Not Applicable \\
\hline Others & $\begin{array}{l}\text { St. John's wort } \\
\text { (Hypericum perforatum), modafinil }\end{array}$ & Not Applicable \\
\hline \multicolumn{3}{|l|}{ CYP3A4 inhibitors } \\
\hline Antibiotics & $\begin{array}{l}\text { Clarithromycin, erythromycin, } \\
\text { troleandomycin }\end{array}$ & 7 days \\
\hline Antifungals & $\begin{array}{l}\text { Itraconazole, ketoconazole, } \\
\text { fluconazole (>150 mg/d), } \\
\text { voriconazole }\end{array}$ & \\
\hline $\begin{array}{l}\text { Antiretrovirals, } \\
\text { protease inhibitors }\end{array}$ & $\begin{array}{l}\text { Delavirdine, nelfinavir, } \\
\text { amprenavir, ritonavir, indinavir, } \\
\text { saquinavir, lopinivir }\end{array}$ & \\
\hline $\begin{array}{l}\text { Calcium channel } \\
\text { blockers }\end{array}$ & Verapamil, diltiazem & \\
\hline Antidepressants & Nefazodone, fluvoxamine & \\
\hline Gl agents & Cimetidine, aprepitant & \\
\hline \multirow[t]{2}{*}{ Others } & Grapefruit, grapefruit juice & \\
\hline & Amiodarone & 6 months \\
\hline \multicolumn{3}{|l|}{ Miscellaneous } \\
\hline Antacids & Mylanta ${ }^{\oplus}$, Maalox $^{\oplus}$, Tums $^{\oplus}$, Rennie ${ }^{\circledast}$ & $\begin{array}{l}1 \mathrm{~h} \text { before and } \\
\text { after dosing }\end{array}$ \\
\hline $\begin{array}{l}\text { Herbal or dietary } \\
\text { supplements }\end{array}$ & $\begin{array}{l}\text { Ginkgo biloba, grape seed, } \\
\text { valerian, ginseng, echinacea, } \\
\text { evening primrose oil }\end{array}$ & 14 days \\
\hline
\end{tabular}

lapatinib will be initiated 14 days prior to radiation therapy (lead-in period) and continued for the duration of radiation therapy. Patients will be instructed not to take lapatinib with grapefruit or grapefruit juice. Moreover, patients will be instructed that if they vomit any time after taking a dose, they must not "make it up" with an extra dose, but instead resume dosing with the next scheduled daily dose. Any missed dose will not be replaced; the dosing should resume with the next scheduled daily dose. Patients will be required to return all used, unused, and/or partially used bottles of lapatinib, and the number of remaining tablets will be documented and recorded to assess compliance.

\section{Capecitabine}

Capecitabine, 150-mg tablets and 500-mg tablets, will be self-administered at a dose of $825 \mathrm{mg} / \mathrm{m}^{2}$ (dose rounded to the nearest possible combination of the available tablet formulations) twice daily, within 30 minutes of a meal, with water, for the duration of radiotherapy. Patients will be required to return all used, unused, and/ or partially used blister packs of capecitabine at the next study visit. The number of tablets remaining will be documented and recorded to assess compliance.

\section{Radiation therapy}

Patients will be treated with megavoltage energy to a total treatment dose of 50.4 Gy to the clinical target volume. This treatment will be administered in 1.8-Gy daily fractions, 5 days per week. The treatment technique will be decided by a senior radiotherapist with expertise in rectal cancer treatment. The following techniques will be available: 3D conformal external beam radiation (3D-RT), intensity modulated radiotherapy (IMRT), and volume modulated arc therapy (VMAT). The choice of technique will depend on the need to protect the organs at risk for increased toxicity with radiation treatment based on the planning CT scan. The planning CT scan will image the lower abdomen and pelvis, from first lumbar vertebra to $3 \mathrm{~cm}$ below the buttocks soft tissue projection, with 2.5$\mathrm{mm}$-thick slices. For both the planning CT scan and treatment, the patient will be in a supine position, with head and knee support and hands resting on the thorax with the bladder comfortably full [41]. Volume delineation and dose prescription will be done according to the guidelines set forth by International Commission on Radiation Units (ICRU) Reports 50 and 62 for 3D-RT and ICRU Report 83 for IMRT or VMAT. The primary tumor and regional lymph nodes will be included in the treatment volumes [42-44]. The following volumes will be considered: gross tumor volume (GTV), which includes the rectal tumor and suspicious lymph nodes; clinical tumor volume 45 Gy (CTV45), which includes the rectal tumor and the regional lymph nodes (delimited superiorly by the common 
iliac arteries bifurcation and inferiorly by the obturator canal); the planned target volume 45 Gy (PTV45), which includes the CTV45 with a 1-cm external margin except when that margin is the skin; the clinical tumor volume 50.4 Gy (CTV50.4), which includes the GTV and the entire mesorectum and presacral region at involved levels and a 2-cm cephalad and caudal margin beyond any suspicious lymph node, and for tumors located at the anorectal junction, the CTV50.4 will include the GTV and a 1-cm anterolateral and posterior margin in addition to the above mentioned cephalad and caudal margin; and the planned target volume 50.4 Gy (PTV50.4), which includes the CTV50.4 with a 1-cm margin, except at the skin.

During treatment planning the following organs will be considered at risk of unacceptable toxicity during pelvic radiation: bladder, small intestine, and femurs (head of femur). To protect against this risk, during treatment planning, dose-volume histograms for these organs will be independently calculated to ensure that the dose constraints set forth in Table 3 for each of the organs at risk are abided by [45-47].

\section{Surgery}

For all included patients, surgical treatment will be performed 7 to 8 weeks after the end of radiation therapy. The choice of surgical technique will depend on preoperative assessment by a senior surgeon trained in rectal cancer surgery. Total mesorectum excision will be recommended.

\section{Management of trial interventions}

During treatment, all patients will be observed weekly to assess toxicity. Patients are allowed to receive full supportive care therapies concomitantly during the study. Summary recommendations for dose modification due to treatment emergent adverse events are defined and outlined in Table 4. No individual lapatinib dose reduction or dose escalation will be allowed to manage treatmentrelated adverse events. In the event of excess toxicity at a dose level of $1250 \mathrm{mg} / \mathrm{d}$ of lapatinib, the trial will be stopped and restarted with lapatinib dosed at $1000 \mathrm{mg} / \mathrm{d}$. If a patient requires holding of radiation treatment for 2

Table 3 Dose-volume constraints

\begin{tabular}{ll}
\hline Organ at risk & Dose restriction \\
\hline Bladder & V40 $<50 \%$ \\
& V45 $<30 \%$ \\
Small intestine & No more than 180 cc above 35 Gy \\
& No more than 100 cc above 40 Gy \\
& No more than 65 cc above 45 Gy \\
Femoral head & V25 $<45 \%$ \\
& V40 $<40 \%$ \\
& V50 $<10 \%$ \\
\hline
\end{tabular}

or more weeks to manage any adverse event, radiation treatment will be discontinued. In case of radiation treatment interruption, the total dose will be adjusted according to a biological equivalent dose calculation [48]. Any of the study interventions (lapatinib, capecitabine, and radiation therapy) may be discontinued if a patient experiences treatment emergent adverse events judged by the investigator to outweigh its benefits. Treatment with the study drug may be discontinued for any of the following reasons: protocol violation, study termination by the sponsor, loss to follow-up, or death. Additionally patients may discontinue therapy at any time.

Patients who discontinue any of the study drugs prior to surgical treatment may remain in the study and undergo radiation therapy (if this was not the cause of study drug discontinuation) and surgical treatment as per protocol. Patients who discontinue study drug due

Table 4 Summary of dose modification guidelines

\begin{tabular}{|c|c|c|c|}
\hline Adverse event (CTCAE v4.0) & Lapatinib & Capecitabine & Radiation \\
\hline \multicolumn{4}{|c|}{ Hematology: neutrophils or platelets } \\
\hline Grade -2: & Continue & Hold 7 days $^{a}$ & Continue \\
\hline Grade $\geq 3:$ & & Hold 7 days $^{a}$ & Hold $^{b}$ \\
\hline \multicolumn{4}{|l|}{ Dermatology: rash } \\
\hline Grade -3: & Hold 7 days $^{a}$ & Not applicable & \\
\hline \multicolumn{4}{|l|}{ Dermatology: irradiated skin } \\
\hline Grade $\geq 3:$ & Not applicable & & Hold $^{b}$ \\
\hline \multicolumn{4}{|c|}{ Dermatology: palmar-plantar erythrodysesthesia } \\
\hline Grade $\geq 2$ : & Not applicable & Hold 7 days $^{a}$ & Not applicable \\
\hline \multicolumn{4}{|l|}{ Gastroenteroloy: diarrhea } \\
\hline Grade $\geq 3:$ & Discontinue & Hold $^{a}$ & Hold $^{b}$ \\
\hline \multicolumn{4}{|l|}{ Respiratory: pneumonitis } \\
\hline Grade $\geq 3:$ & Discontinue & & \\
\hline \multicolumn{4}{|l|}{ Cardiac dysfunction: } \\
\hline $\begin{array}{l}\text { Asymptomatic LVEF decrease } \\
>20 \% \text { from baseline and } \\
\text { absolute value below the LLN }\end{array}$ & Discontinue & Not applicable & \\
\hline \multicolumn{4}{|l|}{ Grade -3} \\
\hline \multicolumn{4}{|l|}{ Hepatotoxicity: } \\
\hline $\begin{array}{l}\mathrm{ALT}>3 \times \text { ULN AND ALT } \\
<5 \times U L N\end{array}$ & Continue & Not applicable & \\
\hline $\begin{array}{l}\mathrm{ALT}>3 \times \text { ULN AND ALT } \\
>5 \times \text { ULN for } \geq 2 \text { weeks }\end{array}$ & Discontinue & & \\
\hline \multicolumn{4}{|l|}{$\begin{array}{l}\mathrm{ALT}>3 \times \text { ULN AND ALT } \\
<5 \times \text { ULN for }>4 \text { weeks }\end{array}$} \\
\hline \multicolumn{4}{|l|}{$\begin{array}{l}\text { ALT }>3 \times \text { ULN AND bilirubin } \\
>2 \times U L N(>35 \% \text { direct })\end{array}$} \\
\hline \multicolumn{4}{|l|}{$\begin{array}{l}\mathrm{ALT}>3 \times \text { ULN AND ALT } \\
>8 \times U L N\end{array}$} \\
\hline $\begin{array}{l}\text { ALT }>3 \times \text { ULN AND signs } \\
\text { and symptoms of hepatitis } \\
\text { or hypersensitivity }\end{array}$ & & & \\
\hline
\end{tabular}


to a discontinuation of radiation therapy and are deemed surgically eligible will be surgically treated as per local standard of care. Patients who discontinue study drug will not be replaced.

\section{Follow-up}

The end of treatment visit will be performed approximately 14 days after the last dose of radiochemotherapy. The end of study visit will be performed 30 days after the surgical intervention. In the event a patient develops any study-related toxicity, he will be followed until its resolution or clinical stabilization.

\section{Tumor specimen management}

Biopsy fragments will be immediately placed in buffered formalin and allowed to be fixed for 24-48 h. Then, the specimen will be processed overnight for paraffin inclusion. Four-micrometer-thick sections will be obtained from each block, at four levels, and stained with hematoxylin and eosin for histopathological assessment.

The surgical specimen will be delivered to the pathologist fresh and unopened immediately after resection. After inspection and recording of any area of perforation, the non-peritonealized resection margin should be carefully inked. The specimen should then be opened from its anterior surface, excepting the area of the tumor, which should be left unopened to allow for adequate documentation of the circumferential radial margin (CRM). A foam or absorbent paper will then be passed through the residual lumen at the tumor site to aid the penetration of the fixative. The specimen will be allowed to fix in buffered formalin for 24-48 h before further dissection, description, and sampling. The specimen will be sliced transversely at $3-4 \mathrm{~mm}$ intervals within a range of $3 \mathrm{~cm}$ from the tumor, both proximally and distally. At a minimum, tissue sampling should include four blocks of the tumor, disclosing the deepest level of invasion, involvement of serosal surface or any adjacent organ, and extramural vein invasion; a block from the closest distance of the tumor or extramural deposit or tumor in a lymph node to the non-peritonealized resection margin; a block from the proximal and the distal margins (one each); a block from the transition between the tumor and adjacent normal mucosa; a block from normal mucosa; all lymph nodes identified; any other macroscopic abnormality. If no definite residual tumor can be recognized, the whole scarred area will be blocked for histopathological evaluation. Assessment of pathologic response will be done according to EURECA-CC2 guidelines [49].

\section{RAS mutational analysis}

Hematoxylin and eosin stained slides will be carefully reviewed by a pathologist to delimit areas with $>50 \%$ tumor cells, and adjacent tissue sections will be obtained from formalin-fixed paraffin-embedded tissue for DNA extraction. Specific primers will be used to amplify a 92-bp amplicon of KRAS (exon 2, 3, or 4) or NRAS (exon 2, 3, or 4), which will then be analyzed by high resolution melting (HRM) to screen for mutations. In cases positive by HRM, KRAS exons 2 (codons 12 and 13), 3 (codons 59 and 61), and 4 (codons 117 and 146) and NRAS exons 2 (codons 12 and 13), 3 (codons 59 and 61), and 4 (codons 117 and 146) will be identified by $\mathrm{SNaPshot,} \mathrm{which} \mathrm{is} \mathrm{based} \mathrm{on} \mathrm{the} \mathrm{dideoxy} \mathrm{single-base}$ extension of an unlabeled oligonucleotide primer. Fragment analyses will be done by capillary electrophoresis. Only patients without KRAS (exon 2, 3, or 4) or NRAS (exon 2, 3, or 4) mutations will be enrolled.

\section{Biomarker assessment}

EGFR gene amplification and HER-2 gene amplification will be analyzed by fluorescence in situ hybridization on 4- $\mu \mathrm{m}$ thick sections of a representative formalin-fixed paraffin-embedded tissue block. Commercial probes will be used: a test probe that targets EGFR and a control probe for chromosome 7 centromere (CEP7) (Vysis), and a test probe that targets HER-2 and a control probe for chromosome 17 centromere (CEP17) (QBiogene). Slides and probes will be codenatured, and hybridization will take place overnight. After post-hybridization washes, slides will be counterstained with DAPI. Fluorescent images will be sequentially captured with a Cohu 4900 CCD camera, using an automated filter wheel coupled to a Zeiss Axioplan fluorescence microscope and a CytoVision system. Gene amplification will be scored when a ratio EGFR/CEP7 or HER-2/CEP17 is greater than or equal to 2.0 in a minimum of 60 cancer cell nuclei.

EGFR, protein kinase (AKT), and mitogen-activated protein kinase (MAPK) phosphorylation status and Ki67 will be assessed by immunohistochemical analysis of paraffin-embedded, formalin-fixed tumor tissue. Four- $\mu \mathrm{m}$ sections will be cut and placed in silanized slides. Immunostaining will be performed using a polymer method (BrightVision Poly-HRP-Anti Ms/Rb/Rt IgG, ImmunoLogic, Duiven, The Netherlands). After dewaxing the sections, endogenous peroxidase activity will be inhibited with freshly prepared $0.5 \%$ hydrogen peroxide in distilled water for $20 \mathrm{~min}$. Antigen retrieval will be performed with EDTA buffer, $\mathrm{pH} 8$, for 40 minutes. Incubation with primary antibodies for phosphorylated EGFR (clone Tyr1173 e 53A5), phosphorylated AKT (clone Ser473 e D9E), phosphorylated MAPK (clone Erk1/2,Thr202 e Tyr204), and Ki-67 (clone MIB-1, Dako, Glostrup, Denmark) will be performed overnight at $4{ }^{\circ} \mathrm{C}$, at dilution $1: 250$, in $1 \%$ bovine serum albumin (BSA) in phosphate-buffered saline (PBS). All incubations will be performed in a humidified chamber. Sections will be developed with a peroxidase substrate solution (0.05\% 3,3-diaminobenzidine 
tetrahydrochloride, $0.01 \% \mathrm{H}_{2} \mathrm{O}_{2}$ in $\mathrm{PBS}$ ), counterstained with hematoxylin, dehydrated, and mounted. Appropriate positive and negative controls will be used. Assessment of immunoexpression will be performed by light microscopy at $\times 400$ magnification by a senior pathologist.

\section{Statistical considerations and quantitative analyses Study endpoints}

The primary endpoint is pathologic complete remission (pCR). Pathologic complete remission will be determined on the surgical specimen and defined according to the guidelines recommended by the EURECA-CC2 consensus [2] by a pathologist blinded to the treatment arm.

The secondary endpoint will be the safety profile of the combination lapatinib-capecitabine and radiation therapy in the preoperative setting of patients with resectable rectal cancer assessed by the identification of adverse events and serious adverse events coded with the National Cancer Institute's Common Terminology Criteria for Adverse Events (CTCAE), version 4.0. An adverse event is defined as any undesirable event associated with the use of a drug, whether or not considered drug related, and includes any side effect, injury, toxicity, or sensitivity reactions, or any undesirable clinical or laboratory event not normally observed in the patient. The following definition of serious adverse event is used: any adverse event that results in death, or is life-threatening, or results in persistent or significant disability or incapacity, requires or prolongs hospitalization, or results in a congenital abnormality or birth defect, or results in any other medically important condition, or an adverse event as a result of an overdose, or is a drug overdose (defined as $\geq 25 \%$ increase in dose over the protocol-specified dose or more doses are given in a cycle).

Additionally the following exploratory endpoints will be addressed: frequency of and predictive value for the primary endpoint of EGFR amplification status, HER-2/neu amplification status, and predictive value for the primary endpoint of inhibition of EGFR and EGFR downstream proteins on the 14th day of treatment in patients allocated to arm A (MAPK, AKT).

\section{Determination of sample size}

The trial's objective is to show the superiority of the lapatinib and capecitabine combination (arm A) over the standard capecitabine (arm B). Thus, the null hypothesis is defined as $\mathrm{H}_{0}: \mathrm{pB} \geq \mathrm{pA}$, where $\mathrm{pA}$ and $\mathrm{pB}$ are the probabilities of a pCR for arms $A$ and $B$, respectively.

The expected sample size calculations were conducted in the context of a Bayesian adaptive randomization design, with stopping rules for early evidence of efficacy/ futility and evidence of toxicity. These calculations are based on simulated trial results, which require presetting maximum and minimum total sample size, minimum number of patients fairly randomized to each arm, minimum probability of randomization for each arm, prior distributions for the treatment effect, and stopping rules for efficacy/futility.

We chose a maximum of 80 patients, based on the feasibility of patient's accrual during the proposed study period. A minimum of 30 patients should be enrolled in the study before stopping the trial due to early evidence of efficacy/inefficacy. However, accrual may stop before the enrollment of 30 patients if there is evidence of toxicity, as defined below. The first 20 patients enrolled in the study will be fairly (non-adaptively) randomized to both arms. During the adaptive randomization phase, the minimum probability of randomization for each arm is set at 0.1 .

For arm A, a non-informative prior for treatment effect, i.e., $\mathrm{pA} \sim$ Uniform $(0,1)$, and for arm $\mathrm{B}$, a moderate informative prior for treatment effect $\mathrm{pB} \sim \operatorname{Beta}(1.2,8.8)$ are used, as shown in Figs. 1 and 2, respectively.

The trial will be stopped for efficacy if the probability of one arm being superior to the other is greater than 0.95 and for futility if the same probability is less than 0.05. Additionally, the trial stops for futility if the probability of arm A having an effect greater than 0.2 is less than 0.05 .

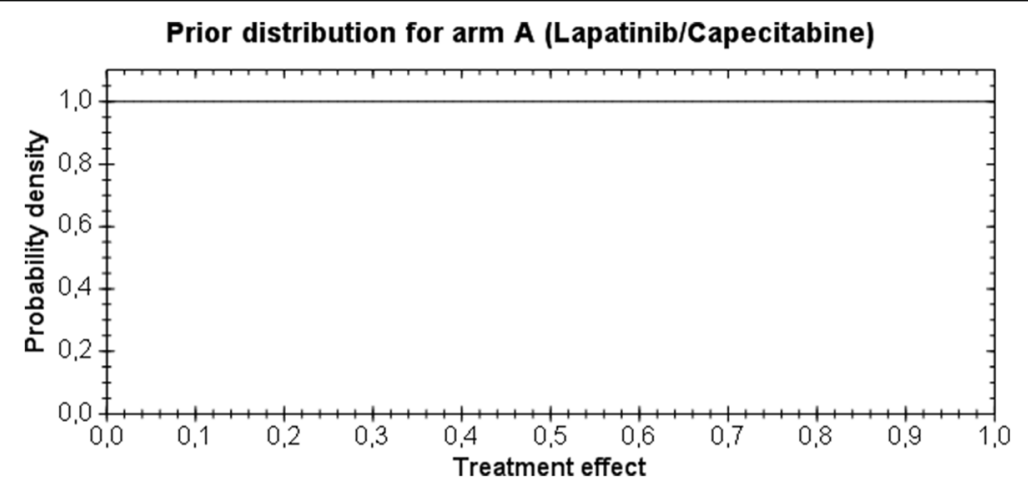

Fig. 1 Prior distribution for arm A 


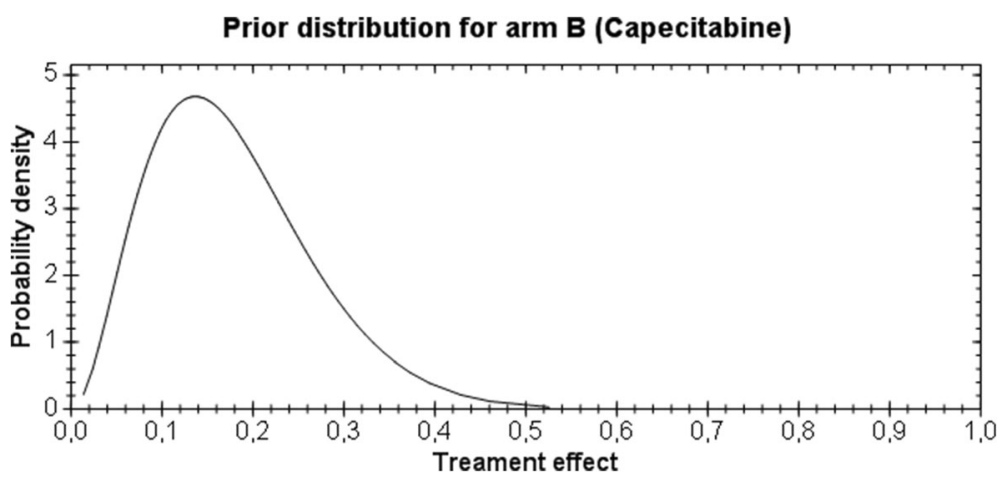

Fig. 2 Prior distribution for arm B

Based on the previous setup, expected sample sizes were computed for three different scenarios, based on 10,000 simulations of trial results:

I. $\mathrm{pA}=0.30$ and $\mathrm{pB}=0.12$

II. $\mathrm{pA}=0.25$ and $\mathrm{pB}=0.12$

III. $\mathrm{pA}=0.12$ and $\mathrm{pB}=0.12$

For scenario I, the expected sample size is 50 with 34 patients enrolled in arm A and 16 in arm B. The probability of choosing arm A as superior is $82 \%$ (equivalent to the power concept in the frequentist framework), and the probability of early stop due to efficacy is $78 \%$. The expected trial length is 13 months. For scenario II, 56 patients are expected to enroll in the trial with $37 \mathrm{pa}$ tients in arm A and 29 in arm B. The probability of identifying arm $\mathrm{A}$ as superior is $63 \%$ (equivalent to the power concept in the frequentist framework), and the probability of early stop due to efficacy is $64 \%$. The expected trial length is 15 months. Finally, if no differences exist between the two arms (scenario III), the expected sample size is 60 (30 each arm). The probability of choosing one of the arms as superior is $12 \%$ (equivalent to the probability of type I error in the frequentist framework). The expected trial length is 16 months.

\section{Randomization and stratification}

The first 20 patients will be enrolled to arm A or B based on a pretrial 1:1 random allocation list generated by the trial statistician and made available to the research team in sealed envelopes. After the first 20 patients, the statistician will provide the randomization result for each patient according to the adaptive design. If a patient discontinues participating in the study, his/her randomization code will not be reused, and the patient will not be allowed to re-enter the study.

\section{Quantitative analysis}

The following patient populations will be considered for analysis:
- Intention-to-treat (ITT) population: the ITT population is defined as all patients who are randomized. Patients in this population will be analyzed according to the treatment they were randomized to receive, regardless of any errors of dosing.

- Safety population: the safety population will be assessed per treatment arm. For arm A it includes all patients who have completed at least one administration of lapatinib, irrespective of starting treatment with capecitabine (safety population in arm A). For arm B it includes all patients allocated to arm B and who completed at least one administration of capecitabine (safety population in arm B).

- Per protocol (PP) population: the PP population includes all patients who started the allocated treatment and have been submitted to surgical treatment.

- Exploratory subgroup analysis (ESA) population: the ESA population includes patients with complete data for the exploratory biomarkers who did not have any major protocol violation.

All available efficacy and safety data will be included in data listings and tabulations. However, for primary endpoint analysis any missing information regarding the endpoint (loss to follow-up or death prior to surgery and refusal to undergo surgery) will be assumed as absence of pathologic complete response. The impact of this imputation will be tested with a sensitivity analysis. For assessment of the stopping rule of toxicity, loss to follow-up (after all efforts made to assess the reason for it have failed) will be considered to be due to a serious adverse event.

Demographic and baseline characteristics including gender, age, weight, height, clinical stage (American Joint Committee on Cancer's 7th edition cTN status [40]), EGFR and HER-2 amplification status, and other parameters, as appropriate, will be summarized by treatment groups using descriptive statistics. 
The proportion of $\mathrm{pCR}$ of each arm will be described and the efficacy of one arm over the other estimated by the posterior probability of superiority and its $95 \%$ credible interval. The trial will be stopped for efficacy if the posterior probability of one arm being superior to the other is greater than 0.95 and for futility if the same probability is less than 0.05 . Additionally, the trial stops for futility if the probability of arm A having an effect greater than 0.2 is less than 0.05 . The posterior probability of superiority of arm A over arm B will be continuously calculated after the 30th patient outcome is known.

Adverse events will be described as absolute number of patients presenting any given adverse event during treatment, its proportion, and respective $95 \%$ credible intervals. Data will also be summarized as total number of patients presenting at least one serious adverse event and its proportion. For analysis of serious adverse events, a summary posterior probability of a serious adverse event and its $95 \%$ credible interval will be calculated. Patient accrual for arm A will be stopped for toxicity if there is evidence of a serious adverse event posterior probability (SAEPP) greater than $25 \%$. A conservative threshold of 0.5 will be used for the posterior probability of toxicity, meaning that the trial stops with moderate evidence (50\%) of SAEPP $>25 \%$ for arm A. A non-informative prior (Beta $(1,1))$ for the proportion of toxic events was chosen. The boundary at each step is plotted in Fig. 3. Should this toxicity boundary be identified for arm A, accrual to the trial is interrupted for excess toxicity at a lapatinib dose level of $1250 \mathrm{mg} / \mathrm{d}$. The trial will then restart with lapatinib at a dose level of $1000 \mathrm{mg} / \mathrm{d}$ in arm A, and the data from patients previously randomized to arm $B$ will be used to adjust the prior distribution of $\mathrm{pB}$ necessary for random treatment allocation in the Bayesian framework previously defined. In this latter setting, should the toxicity boundary be crossed, accrual to the trial is stopped for toxicity and the combination of lapatinib with capecitabine as radiosensitizer will be considered too toxic for further study.

Biomarkers will be described as the proportion of patients with the given biomarker present in the respective arm and their $95 \%$ credible intervals. Identification of predictive biomarkers will be performed through logistic regression analysis. The model will test the association between activation of EGFR pathway at baseline and pCR (between group analyses) and the discriminatory ability of its inhibition on pCR on arm A (within group analysis). The following biomarkers will be included in the model: EGFR amplification; HER-2/neu amplification; MAPK phosphorylation status; AKT phosphorylation status; EGFR phosphorylation status at baseline (between group analysis) and at baseline and on day 14 biopsy specimens for patients in arm A (within group analysis). Odds ratios will be presented with their $95 \%$ credible intervals, and the c-statistics (area under the receiver operating characteristic curve) will be computed.

\section{Ethical considerations}

The protocol has been written, and the study will be conducted according to the ICH Harmonized Tripartite Guideline on Good Clinical Practice. All patients will be informed about the aims of the study, trial procedures, possible hazards and adverse events, the mechanism of treatment allocation, and procedures to ensure patient data confidentiality before enrollment.

The trial was submitted for ethical review by Portugal's National Ethics Committee for Clinical Research and was approved on 7 February 2014, deliberation number 2014RP-02-11.

During the trial, if a protocol amendment is deemed necessary and may significantly affect the safety of patients, the scope of investigation, or the scientific quality of the study, a prior favorable opinion from Portugal's National Ethics Committee for Clinical Research and approval from the Portuguese National Authority of

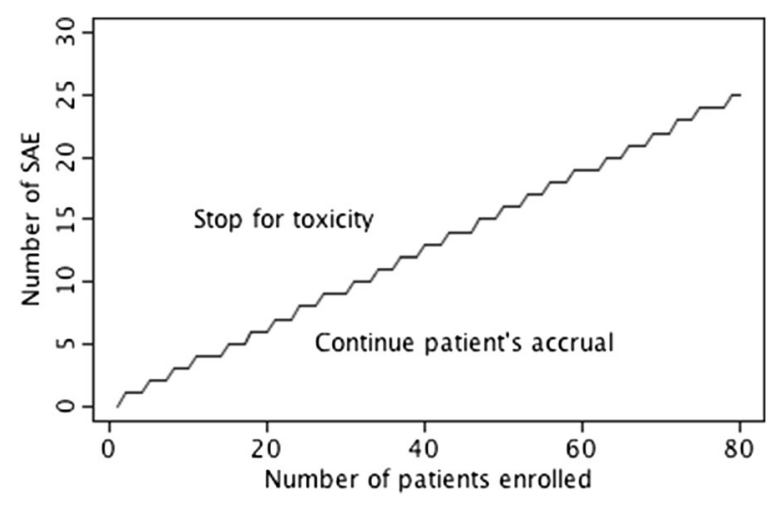

Fig. 3 Boundary for early stop due to toxicity 
Medicines and Health Products will be sought before implementation. If a protocol amendment affects only administrative aspects of the study, both the Portuguese National Ethics Committee for Clinical Research and Portuguese National Authority of Medicines will be notified of such administrative changes, but prior approval will not be procured.

Clinical trial insurance has been taken out according to Portuguese law by the sponsor.

\section{Discussion}

To our knowledge, this is the first trial to assess the efficacy of dual inhibition of EGFR and HER-2 as a radiosensitizer in resectable rectal cancer. The trial is designed to include an enriched population of patients with KRAS and NRAS wild-type rectal cancer due to evidence of lack of benefit from anti-EGFR strategies in KRAS or NRAS mutated colorectal tumors. However, the role of KRAS or NRAS mutations in localized rectal cancer is uncertain. There is no information on whether this subgroup of rectal cancer patients have a different prognosis compared to patients with activating mutations nor if absence of mutation in KRAS or NRAS is predictive of response to standard radiochemotherapy. Thus, a randomized trial with an active comparator was needed to answer this question. The choice of a randomized adaptive Bayesian design allows an optimized sample size as well as a concurrent comparator. This design optimizes the trial's sample size and protects eligible patients from randomization to a weakly performing arm, thus improving our ability to decide whether further study of lapatinib in this setting is warranted [50,51].

The primary objective of the trial is to show that lapatinib-capecitabine radiochemotherapy is superior to capecitabine radiochemotherapy. The choice of complete pathologic response as the primary outcome measure allows such interpretation since it is a feasible and reproducible short-term endpoint and is reported to be associated with improved cancer-specific survival [7]. The choice to use full doses for each treatment component maximizes the probability of treatment response. As with any novel treatment combination, tolerability will be an important issue. The experimental arm has never been studied in this setting. Therefore, we have integrated into the Bayesian design a specific stopping rule to address this. This rule was designed to reproduce the performance of classic phase I trials, which are designed to explore the maximum tolerated dose of a given agent or combination while protecting as many patients as possible from excessive risks. The main anticipated toxicity is diarrhea; therefore, mandatory pre-emptive antidiarrheal medication will be recommended for every patient. Should excess toxicity be an issue with a standard dose of lapatinib, we have stipulated a reset of the trial with a lower dose of lapatinib. However, this should not impact the assessment of clinical value of this combination, as the lower dose level of lapatinib is still associated with a $\mathrm{C}_{\min }$ above that which is required for antitumor effect. Additionally, the incorporation of a lead-in period of lapatinib together with tumor sampling before and after the 2-week lead-in period will provide further information on the pharmacodynamics of lapatinib and direct evidence of on-target effect.

This trial includes an exploratory biomarker program. This was designed to better understand the role that EGFR inhibitors and lapatinib in particular play in this setting and to identify potentially predictive biomarkers for response.

In conclusion, the LaRRC trial will inform on the role of dual inhibition of EGFR and HER-2 in the neoadjuvant treatment of resectable rectal cancer. The choice of a Bayesian adaptive randomization together with an initial lead-in treatment phase with lapatinib in the experimental arm provides the best chance to maximize the information collected while effectively balancing the risks and potential benefits for eligible patients.

\section{Trial status}

The trial is currently recruiting.

\begin{abstract}
Abbreviations
3D-RT: 3D conformal external beam radiation; CRM: Circumferential radial margin; CTV45: Clinical tumor volume 45 Gy; CTV50.4: Clinical tumor volume 50.4 Gy; CTCAE: Common Terminology Criteria for Adverse Events; DPD: Dihydropyrimidine dehydrogenase; EGFR: Epidermal growth factor receptor; ESA: Exploratory subgroup analysis; GTV: Gross tumor volume; HRM: High resolution melting; IMRT: Intensity modulated radiotherapy; ITT: Intention to treat; pCR: Pathologic complete remission; PP: Per protocol; PTV45: Planned target volume 45 Gy; PTV50.4: Planned target volume 50.4 Gy; SAEPP: Serious adverse event posterior probability; ULN: Upper limit of normality; VMAT: Volume modulated arc therapy
\end{abstract}

\section{Acknowledgements}

This section is not applicable.

\section{Funding}

The trial is funded by its sponsor: Instituto Português de Oncologia do Porto, EPE. GlaxoSmithKline provided lapatinib free of charge.

The sponsor was not involved in the design of the study and it will provide all necessary means to implement the trial. Trial data analysis will be performed without direct intervention of the sponsor by the trial statistician. The publication of any trial results will be written by the investigators, led by the Principal Investigator. Prior to any study data publication, results will be submitted for review to the Instituto Português de Oncologia do Porto, EPE, and advanced knowledge of all publications was agreed to be given to GlaxoSmithKline.

The sponsor's contact details are as follows: IPO Porto FG, EPE

Rua Dr. António Bernardino de Almeida

4200-072 Porto, Portugal

Tel: +351225084000

Fax: +351225084008

e-mail: clinicalstudies@ipoporto.min-saude.pt

web address: www.ipoporto.min-saude.pt

Availability of data and materials

This section is not applicable. 


\section{Authors' contributions}

NS conceived the trial, participated in the trial's design, and wrote the manuscript. OS, LLS, RH, MRT, and MDR participated in the trial's design. ATP participated in the trial's design, statistical analysis design, and trial operating characteristics assessment. All authors read and approved the final manuscript.

\section{Competing interests}

This trial was made possible by GlaxoSmithKline, who provided the investigational medicinal product free of charge. No non-financial competing interest is declared. The authors declare that they have no competing interests.

\section{Consent for publication}

This section is not applicable.

\section{Ethics approval and consent to participate}

The trial was submitted for ethical review by Portugal's National Ethics Committee for Clinical Research and was approved on 7 February 2014, deliberation number 2014-RP-02-11.

\section{Author details}

'Medical Oncology Department, IPO Porto FG, EPE, Rua António Bernardino de Almeida, 4200-072 Porto, Portugal. ${ }^{2}$ Radioncology Department, IPO Porto FG, EPE, Rua António Bernardino de Almeida, 4200-072 Porto, Portugal. ${ }^{3}$ Surgical Oncology Department, IPO Porto FG, EPE, Rua António Bernardino de Almeida, 4200-072 Porto, Portugal. ${ }^{4}$ Department of Pathology, IPO Porto FG, EPE, Rua António Bernardino de Almeida, 4200-072 Porto, Portugal. ${ }^{5}$ Department of Pathology and Molecular Immunology, Abel Salazar Institute of Biomedical Sciences - University of Porto, Rua de Jorge Viterbo Ferreira, no. 228, 4050-313 Porto, Portugal. ${ }^{6}$ Genetics Department, IPO Porto FG, EPE, Rua António Bernardino de Almeida, 4200-072 Porto, Portugal. ${ }^{7}$ Biomedical Sciences Institute Abel Salazar (ICBAS), University of Porto, Porto, Portugal. ${ }^{8}$ Gastroenterology Department, IPO Porto FG, EPE, Rua António Bernardino de Almeida, 4200-072 Porto, Portugal. ${ }^{9}$ CINTESIS - Center for Health Technology and Services Research, Centro de Investigação Médica, Faculdade de Medicina da Universidade do Porto, Rua Dr. Plácido da Costa, $s / n, 4200-450$ Porto, Portugal. ${ }^{10}$ Screening and Test Evaluation Program (STEP), Sydney School of Public Health, The University of Sydney, Sydney, NSW 2006, Australia.

Received: 11 June 2015 Accepted: 23 August 2016 Published online: 21 September 2016

\section{References}

1. Ferlay J, Autier P, Boniol M, Heanue M, Colombet M, Boyle P. Estimates of the cancer incidence and mortality in Europe in 2006. Ann Oncol. 2007; 18(3):581-92. doi:10.1093/annonc/mdl498.

2. Curado MP, Edwards B, Shin HR, Storm H, Ferlay J, Heanue M, Boyle P, editors. Cancer incidence in five continents, vol. IX. IARC scientific publications. Lyon: IARC (International Agency for Research on Cancer); 2009.

3. Coleman MP, Quaresma M, Berrino F, Lutz JM, De Angelis R, Capocaccia R, et al. Cancer survival in five continents: a worldwide population-based study (CONCORD). Lancet Oncol. 2008;9(8):730-56. doi:10.1016/S1470-2045(08)70179-7.

4. Kapiteijn E, Putter $\mathrm{H}$, van de Velde CJ. Impact of the introduction and training of total mesorectal excision on recurrence and survival in rectal cancer in The Netherlands. Br J Surg. 2002;89(9):1142-9. doi:10.1046/j.13652168.2002.02196.x

5. Wong RK, Tandan V, De Silva S, Figueredo A. Pre-operative radiotherapy and curative surgery for the management of localized rectal carcinoma. Cochrane Database Syst Rev. 2007:2:CD002102. doi:10.1002/14651858.CD002102.pub2.

6. Ceelen WP, Van Nieuwenhove Y, Fierens K. Preoperative chemoradiation versus radiation alone for stage $\mathrm{II}$ and III resectable rectal cancer. Cochrane Database Syst Rev. 2009;1:CD006041. doi:10.1002/14651858.CD006041.pub2.

7. Collette $L$, Bosset JF, den Dulk M, Nguyen F, Mineur $L$, Maingon $P$, et al. Patients with curative resection of cT3-4 rectal cancer after preoperative radiotherapy or radiochemotherapy: does anybody benefit from adjuvant fluorouracil-based chemotherapy? A trial of the European Organisation for Research and Treatment of Cancer Radiation Oncology Group. J Clin Oncol. 2007;25(28):4379-86. doi:10.1200/JCO.2007.11.9685.

8. Bosset JF, Collette L, Calais G, Mineur L, Maingon P, Radosevic-Jelic L, et al. Chemotherapy with preoperative radiotherapy in rectal cancer. N Engl J Med. 2006;355(11):1114-23. doi:10.1056/NEJMoa060829.
9. Gerard JP, Conroy T, Bonnetain F, Bouche O, Chapet O, Closon-Dejardin MT, et al. Preoperative radiotherapy with or without concurrent fluorouracil and leucovorin in T3-4 rectal cancers: results of FFCD 9203. J Clin Oncol. 2006; 24(28):4620-5. doi:10.1200/JCO.2006.06.7629.

10. Capecitabine, summary of product characteristics. http://www.ema.europa. eu/docs/en_GB/document_library/EPAR_-_Product_Information/human/ 000316/WC500058151.pdf.

11. Ballonoff A, Kavanagh B, McCarter M, Kane M, Pearlman N, Nash R, et al. Preoperative capecitabine and accelerated intensity-modulated radiotherapy in locally advanced rectal cancer: a phase II trial. Am J Clin Oncol. 2008; 31(3):264-70. doi:10.1097/COC.0b013e318161dbd3.

12. De Paoli A, Chiara S, Luppi G, Friso ML, Beretta GD, Del Prete S, et al. Capecitabine in combination with preoperative radiation therapy in locally advanced, resectable, rectal cancer: a multicentric phase II study. Ann Oncol. 2006;17(2):246-51. doi:10.1093/annonc/mdj041.

13. Dunst J, Debus J, Rudat V, Wulf J, Budach W, Hoelscher T, et al. Neoadjuvant capecitabine combined with standard radiotherapy in patients with locally advanced rectal cancer: mature results of a phase II trial. Strahlenther Onkol. 2008;184(9):450-6. doi:10.1007/s00066-008-1751-4.

14. Marsh Rde W, George TJ, Siddiqui T, Mendenhall WM, Zlotecki RA, Grobmyer S, et al. A phase II trial of neoadjuvant capecitabine combined with hyperfractionated accelerated radiation therapy in locally advanced rectal cancer. Am J Clin Oncol. 2010;33(3):251-6. doi:10.1097/COC. Ob013e3181a650e8.

15. Velenik V, Anderluh F, Oblak I, Strojan P, Zakotnik B. Capecitabine as a radiosensitizing agent in neoadjuvant treatment of locally advanced resectable rectal cancer: prospective phase II trial. Croat Med J. 2006;47(5):693-700.

16. Hofheinz RD, Wenz F, Post S, Matzdorff A, Laechelt S, Hartmann JT, et al. Capecitabine (Cape) versus 5-fluorouracil (5-FU)-based (neo-)adjuvant chemo-radiotherapy (CRT) for locally advanced rectal cancer (LARC): longterm results of a randomized, phase III trial. Onkologie. 2011;34:199.

17. Salomon DS, Brandt R, Ciardiello F, Normanno N. Epidermal growth factorrelated peptides and their receptors in human malignancies. Crit Rev Oncol Hematol. 1995;19(3):183-232.

18. Dahabreh IJ, Terasawa T, Castaldi PJ, Trikalinos TA. Systematic review: antiepidermal growth factor receptor treatment effect modification by KRAS mutations in advanced colorectal cancer. Ann Intern Med. 2011;154(1):37-49. doi:10.1059/0003-4819-154-1-201101040-00006

19. Douillard JY, Siena S, Cassidy J, Tabernero J, Burkes R, Barugel M, et al. Randomized, phase III trial of panitumumab with infusional fluorouracil, leucovorin, and oxaliplatin (FOLFOX4) versus FOLFOX4 alone as first-line treatment in patients with previously untreated metastatic colorectal cancer: the PRIME study. J Clin Oncol. 2010;28(31):4697-705. doi:10.1200/JCO.2009.27.4860.

20. Karapetis CS, Khambata-Ford S, Jonker DJ, O'Callaghan CJ, Tu D, Tebbutt NC, et al. K-ras mutations and benefit from cetuximab in advanced colorectal cancer. N Engl J Med. 2008;359(17):1757-65. doi:10.1056/NEJMoa0804385.

21. Peeters M, Price TJ, Cervantes A, Sobrero AF, Ducreux M, Hotko $Y$, et al. Randomized phase III study of panitumumab with fluorouracil, leucovorin, and irinotecan (FOLFIRI) compared with FOLFIRI alone as second-line treatment in patients with metastatic colorectal cancer. J Clin Oncol. 2010; 28(31):4706-13. doi:10.1200/JCO.2009.27.6055.

22. Van Cutsem E, Kohne CH, Hitre E, Zaluski J, Chang Chien CR, Makhson A, et al. Cetuximab and chemotherapy as initial treatment for metastatic colorectal cancer. N Engl J Med. 2009;360(14):1408-17. doi:10.1056/ NEJMoa0805019.

23. Van Cutsem E, Lenz HJ, Kohne CH, Heinemann V, Tejpar S, Melezinek I, et al. Fluorouracil, leucovorin, and irinotecan plus cetuximab treatment and RAS mutations in colorectal cancer. J Clin Oncol. 2015;33(7):692-700. doi:10.1200/JCO.2014.59.4812.

24. Quesnelle KM, Grandis JR. Dual kinase inhibition of EGFR and HER2 overcomes resistance to cetuximab in a novel in vivo model of acquired cetuximab resistance. Clin Cancer Res. 2011;17(18):5935-44. doi:10.1158/1078-0432.ccr-11-0370.

25. Yonesaka K, Zejnullahu K, Okamoto I, Satoh T, Cappuzzo F, Souglakos J, et al. Activation of ERBB2 signaling causes resistance to the EGFR-directed therapeutic antibody cetuximab. Sci Transl Med. 2011;3(99):99ra86. doi:10. 1126/scitransImed.3002442.

26. Magne N, Chargari C, Castadot P, Ghalibafian M, Soria JC, Haie-Meder C, et al. The efficacy and toxicity of EGFR in the settings of radiotherapy: focus on published clinical trials. Eur J Cancer. 2008:44(15):2133-43. doi:10.1016/j.ejca.2008.06.029 
27. Milano G, Magne N. Anti-EGFR and radiotherapy. Cancer Radiother. 2004; 8(6):380-2. doi:10.1016/j.canrad.2004.09.002.

28. Czito BG, Willett CG, Bendell JC, Morse MA, Tyler DS, Fernando NH, et al. Increased toxicity with gefitinib, capecitabine, and radiation therapy in pancreatic and rectal cancer: phase I trial results. J Clin Oncol. 2006;24(4): 656-62. doi:10.1200/JCO.2005.04.1749.

29. Marquardt F, Rodel F, Capalbo G, Weiss C, Rodel C. Molecular targeted treatment and radiation therapy for rectal cancer. Strahlenther Onkol. 2009; 185(6):371-8. doi:10.1007/s00066-009-1936-5.

30. Valentini V, De Paoli A, Gambacorta MA, Mantini G, Ratto C, Vecchio FM, et al. Infusional 5-fluorouracil and ZD1839 (gefitinib-Iressa) in combination with preoperative radiotherapy in patients with locally advanced rectal cancer: a phase I and II trial (1839|L/0092). Int J Radiat Oncol Biol Phys. 2008; 72(3):644-9. doi:10.1016/j.jijrobp.2008.01.046.

31. Giannopoulou E, Antonacopoulou A, Floratou K, Papavassiliou AG, Kalofonos HP. Dual targeting of EGFR and HER-2 in colon cancer cell lines. Cancer Chemother Pharmacol. 2009;63(6):973-81. doi:10.1007/s00280-008-0820-9.

32. Zhou Y, Li S, Hu YP, Wang J, Hauser J, Conway AN, et al. Blockade of EGFR and ErbB2 by the novel dual EGFR and ErbB2 tyrosine kinase inhibitor GW572016 sensitizes human colon carcinoma GEO cells to apoptosis. Cancer Res. 2006;66(1):404-11. doi:10.1158/0008-5472.CAN-05-2506.

33. Sambade MJ, Camp JT, Kimple RJ, Sartor Cl, Shields JM. Mechanism of lapatinib-mediated radiosensitization of breast cancer cells is primarily by inhibition of the Raf > MEK > ERK mitogen-activated protein kinase cascade and radiosensitization of lapatinib-resistant cells restored by direct inhibition of MEK. Radiother Oncol. 2009;93(3):639-44. doi:10.1016/j.radonc.2009.09.006.

34. Sambade MJ, Kimple RJ, Camp JT, Peters E, Livasy CA, Sartor Cl, et al. Lapatinib in combination with radiation diminishes tumor regrowth in HER2+ and basal-like/EGFR+ breast tumor xenografts. Int J Radiat Oncol Biol Phys. 2010;77(2):575-81. doi:10.1016/j.jjrobp.2009.12.063.

35. Fields ALA, Rinaldi DA, Henderson CA, Germond CJ, Chu L, Brill KJ, et al. An open-label multicenter phase II study of oral lapatinib (GW572016) as single agent, second-line therapy in patients with metastatic colorectal cancer. J Clin Oncol (Meeting Abstracts). 2005;23(16_suppl):3583.

36. Chu QS, Schwartz G, de Bono J, Smith DA, Koch KM, Versola MJ, et al. Phase I and pharmacokinetic study of lapatinib in combination with capecitabine in patients with advanced solid malignancies. J Clin Oncol. 2007;25(24): 3753-8. doi:10.1200/JCO.2007.11.1765.

37. Midgley RS, Kerr DJ, Flaherty KT, Stevenson JP, Pratap SE, Koch KM, et al. A phase I and pharmacokinetic study of lapatinib in combination with infusional 5-fluorouracil, leucovorin and irinotecan. Ann Oncol. 2007;18(12): 2025-9. doi:10.1093/annonc/mdm366.

38. Siegel-Lakhai WS, Beijnen JH, Vervenne WL, Boot H, Keessen M, Versola M, et al. Phase I pharmacokinetic study of the safety and tolerability of lapatinib (GW572016) in combination with oxaliplatin/fluorouracil/ leucovorin (FOLFOX4) in patients with solid tumors. Clin Cancer Res. 2007; 13(15 Pt 1):4495-502. doi:10.1158/1078-0432.CCR-07-0004

39. Douillard JY, Oliner KS, Siena S, Tabernero J, Burkes R, Barugel M, et al. Panitumumab-FOLFOX4 treatment and RAS mutations in colorectal cancer. N Engl J Med. 2013;369(11):1023-34. doi:10.1056/NEJMoa1305275.

40. Edge SB, American Joint Committee on Cancer. AJCC cancer staging manual. 7th ed. New York: Springer; 2010.

41. Drzymala M, Hawkins MA, Henrys AJ, Bedford J, Norman A, Tait DM. The effect of treatment position, prone or supine, on dose-volume histograms for pelvic radiotherapy in patients with rectal cancer. Br J Radiol. 2009; 82(976):321-7. doi:10.1259/bjr/57848689.

42. Bosset JF, Servagi-Vernat S, Crehange G, Azria D, Gerard JP, Hennequin C. Rectal cancer: the radiation basis of radiotherapy, target volume. Cancer Radiother. 2011;15(6-7):431-5. doi:10.1016/j.canrad.2011.07.236.

43. Myerson RJ, Garofalo MC, El Naqa I, Abrams RA, Apte A, Bosch WR, et al. Elective clinical target volumes for conformal therapy in anorectal cancer: a radiation therapy oncology group consensus panel contouring atlas. Int J Radiat Oncol Biol Phys. 2009;74(3):824-30. doi:10.1016/j.jirobp.2008.08.070.

44. Roels S, Duthoy W, Haustermans K, Penninckx F, Vandecaveye V, Boterberg T, et al. Definition and delineation of the clinical target volume for rectal cancer. Int J Radiat Oncol Biol Phys. 2006;65(4):1129-42. doi:10.1016/j.jrobp.2006.02.050.

45. Kavanagh BD, Pan CC, Dawson LA, Das SK, Li XA, Ten Haken RK, et al. Radiation dose-volume effects in the stomach and small bowel. Int J Radiat Oncol Biol Phys. 2010;76(3 Suppl):S101-7. doi:10.1016/j.jijrobp.2009.05.071.

46. Spalding $A C$, Jee KW, Vineberg $K$, Jablonowski M, Fraass BA, Pan CC, et al. Potential for dose-escalation and reduction of risk in pancreatic cancer using IMRT optimization with lexicographic ordering and gEUD-based cost functions. Med Phys. 2007;34(2):521-9.

47. Viswanathan AN, Yorke ED, Marks LB, Eifel PJ, Shipley WU. Radiation dosevolume effects of the urinary bladder. Int J Radiat Oncol Biol Phys. 2010;76(3 Suppl):S116-22. doi:10.1016/j.jjobp.2009.02.090.

48. Bentzen SM, Joiner MC. The linear-quadratic approach in clinical practice. In: Joiner M, van der Kogel A, editors. Basic clinical radiobiology. 4th ed. London: Hodder Arnold; 2009. p. 120-34.

49. Valentini V, Aristei C, Glimelius B, Minsky BD, Beets-Tan R, Borras JM, et al. Multidisciplinary Rectal Cancer Management: 2nd European Rectal Cancer Consensus Conference (EURECA-CC2). Radiother Oncol. 2009;92(2):148-63. doi:10.1016/j.radonc.2009.06.027.

50. Berry DA. Bayesian clinical trials. Nat Rev Drug Discov. 2006;5(1):27-36. doi:10.1038/nrd1927.

51. Lee JJ, Liu DD. A predictive probability design for phase II cancer clinical trials. Clin Trials. 2008;5:93-106. doi:10.1177/1740774508089279.

\section{Submit your next manuscript to BioMed Central and we will help you at every step:}

- We accept pre-submission inquiries

- Our selector tool helps you to find the most relevant journal

- We provide round the clock customer support

- Convenient online submission

- Thorough peer review

- Inclusion in PubMed and all major indexing services

- Maximum visibility for your research

Submit your manuscript at www.biomedcentral.com/submit
C Biomed Central 